avances

en cienciase ingenierías

II Simposio
Latinoamericano de Aplicaciones Nucleares en la Agricultura

\title{
Determinación de la dosis letal media (DL50) para mutaciones inducidas con rayos gamma en dos variedades de cebada (Hordeum Vulgare L.)
}

\author{
Javier Garófalo Sosa*1, Luis Ponce-Molina', Patricio Noroña Zapata' ', Diego Campaña Cruz' \\ 'Instituto Nacional de Investigaciones Agropecuarias - INIAP, Estación Experimental Santa \\ Catalina, Mejía, Ecuador \\ *Autor para correspondencia / Corresponding autor, email: javier.garofalo@iniap.gob.ec
}

\section{Determination of the medium lethal dose (LD50) for gamma rays induced mutations in two barley varieties (Hordeum Vulgare L.)}

\begin{abstract}
Resumen
La cebada es un cultivo de importancia económica, social y alimentaria en la Sierra ecuatoriana. La cebada es el segundo cereal más cultivado en Ecuador y el cuarto a nivel mundial. La inducción de mutaciones es una técnica de mejoramiento que permite generar variabilidad genética; es por ello, que el INIAP está empleando esta técnica en el cultivo de cebada en busca de germoplasma con características deseables. El objetivo principal de esta investigación fue determinar la dosis letal media (LD50) de irradiación para inducir mutaciones en dos variedades de cebada, usando para ello cinco dosis de rayos gamma (0, 100, 150, 200 y 250 Gy). Los parámetros evaluados fueron: germinación (en invernadero); emergencia, altura de plántula y vigor (en campo). Los resultados mostraron que las variedades respondieron de diferente forma en las distintas dosis empleadas; sin embargo, fue evidente que a medida que la dosis aumentaba, la emergencia, altura de plántula y vigor, se afectaban y mermaban. Se determinó que la dosis letal media se encuentra entre los 150 y 200 Gy. Sin embargo, la dosis que produjo ligeros cambios morfológicos en los materiales y que se la considera como la dosis óptima es la de $150 \mathrm{~Gy}$.
\end{abstract}

Palabras clave: Cereales, DL50, Irradiación, Mejoramiento, Mutantes.

Editado por /

Edited by:

Gabriela Albán

Recibido /

Received:

04/10/2020

Aceptado /

Accepted:

$12 / 11 / 2020$

Publicado en línea / Published online: 01/04/2021

\section{Abstract}

Barley is a crop of economic, social and food importance in the Ecuadorian highlands. Barley is the second most cultivated cereal in Ecuador and the fourth in the world. Induced mutation is an improvement technique that allows generates genetic variability; that's why, INIAP is applying this technique in the barley crop in search of germplasm with desirable characteristics. The main objective of this research was to determine the medium lethal dose (LD50) of irradiation to induce mutations in two barley varieties, using five doses of gamma rays (0, 100, 150, 200 and $250 \mathrm{~Gy})$. The parameters evaluated 
were: germination (in greenhouse); emergency, seedling height and vigor (in the field). The results showed that the varieties responded in different ways to the distinct doses used, however, it was evident that as the dose increased, the emergency, seedling height and vigor were affected and diminished. The medium lethal dose was determined to be between 150 and 200 Gy. However, the dose that produced slight morphological changes in the materials and is considered like the optimal dose is $150 \mathrm{~Gy}$.

Keywords: Cereals, Irradiation, LD50, Mutants, Plant breeding.

\section{INTRODUCCIÓN}

En Ecuador el cultivo de cebada (Hordeum vulgare L.) es el segundo cereal de más amplia distribución en la Sierra ecuatoriana, después del maíz, y se ha difundido entre los 2400 y 3500 m.s.n.m. [1]. En el año 2019, la superficie dedicada al cultivo de cebada fue 9715 hectáreas, distribuidas entre las 10 provincias de la sierra, con una producción de 13450 toneladas, mientras que las importaciones superaron las 72 mil toneladas [2]. Alrededor de 10 mil familias de los sectores marginales de la región andina cultivan este cereal para su alimentación básica, por lo que es considerado un cultivo de seguridad alimentaria [3].

Los problemas que afectan a la cebada son los factores bióticos (enfermedades) y abióticos (condiciones climáticas y de suelo) [4]. En Ecuador, el Instituto Nacional de Investigaciones Agropecuarias (INIAP), es el encargado de buscar soluciones a través del desarrollo de nuevas tecnologías, como la generación de variedades mejoradas que presenten precocidad, resistencia a enfermedades, calidad y alto rendimiento. Para ello, uno de los métodos de mejoramiento utilizado son las mutaciones inducidas [5].

Las mutaciones inducidas han sido usadas para el mejoramiento genético de cultivos reproducidos por semillas como trigo, cebada, entre otros [6]. El uso de las mutaciones en el mejoramiento de especies agronómicas es mejorar uno o varias características de variedades adaptadas a diferentes condiciones edafoclimáticas, como altura de planta [7], tolerancia a factores biótico y abióticos [8].

Las mutaciones inducidas son provocadas por agentes exógenos (físicos, químicos y biológicos) conocidos como mutágenos, que modifican el ADN, causando mutaciones de tipo puntual, deleciones y aberraciones en los cromosomas, provocando cambios importantes en la estructura genómica de los individuos [9]. Los mutágenos físicos, específicamente las radiaciones de tipo gamma, penetran los tejidos vivos [10]. La mutación inducida por mutágenos físicos (rayos $X$ y rayos gamma) es una fuente importante de creación de variación genética al ampliar los acervos genéticos en una especie y lograr asociarlo con la mejora de cultivos [11]. Adicionalmente, los rayos gamma son amigables para el medio ambiente y la humanidad [12].

La magnitud de las alteraciones cromosómicas depende de las dosis e intensidad de los rayos gamma $[11,13]$. En las mutaciones inducidas, lo más importante es determinar 
primero la dosis letal media (DL50) de cada radiación en relación con una especie vegetal en particular [14,15]. Una vez que se determina la DL50, sobre la base de diferentes cambios, especialmente morfológicos, es posible determinar el nivel máximo permisible de exposición a la radiación de un cultivo en particular, ya que existen posibilidades de fracaso de los experimentos si las dosis no son precisas. Las dosis más bajas no crean variabilidad; pero pueden generar hormesis (aumento de división celular, crecimiento y desarrollo en el organismo) [16] y dosis más altas pueden inducir letalidad [17]. Para que un programa de mutaciones inducidas sea eficiente, es necesario generar un apreciable número de variaciones (eventos mutacionales) y poblaciones grandes que permitan un adecuado screening de los genotipos deseados [18].

El acame se lo denomina al desplazamiento del tallo de la planta de su posición vertical, provocando que el cultivo se tienda al nivel del suelo, afectando al rendimiento y calidad de grano de la cebada. Este problema está relacionado con la altura de la planta [19].

El objetivo de esta investigación fue determinar la DL50 de la irradiación con rayos gamma de semillas de dos variedades de cebada, a través de la evaluación en laboratorio y campo; del porcentaje de emergencia, altura y vigor de plántulas.

\section{MATERIALES Y MÉTODOS}

Los ensayos se implementaron en la Estación Experimental Santa Catalina (EESC) del Instituto Nacional de Investigaciones Agropecuarias (INIAP) ubicada en la parroquia Cutuglagua, cantón Mejía, provincia de Pichicha. Latitud: -0,369794, Longitud: $-78,553326^{\circ}$.

Las variedades de cebada que se utilizaron en el estudio fueron: la variedad mejorada INIAP-Cañicapa 2003 de dos hileras (dística) de grano cubierto; y la variedad criolla Rita Pelada de seis hileras (hexástica) de grano desnudo. Las características morfológicas a mejorar en las variedades fueron: para INIAP-Cañicapa 2003 reducir altura de planta y para Rita Pelada la resistencia al acame. Las semillas empleadas fueron de alta calidad física como biológica, con porcentajes de germinación superiores a 90\% y con una humedad del $12 \%$.

Para la irradiación de las semillas se usó rayos gamma, a través de un irradiador categoría I con una fuente de cobalto 60 de la Subsecretaría de Control y Aplicaciones Nucleares (SCAN) del Ministerio de Energía y Recursos Naturales No Renovables (MERNNR). Para calcular los tiempos de irradiación según la dosis, se basó en la calibración del irradiador con un valor $14.13 \mathrm{~Gy} \mathrm{~min}^{-1}$.

Los factores en estudio incluyeron las dos variedades de cebada (INIAP-Cañicapa 2003 y Rita Pelada) y cinco dosis de irradiación (d1: 0 Gy - testigo, d2: 100 Gy, d3: 150 Gy, d4: 200 Gy y d5: 250 Gy), dando en total 10 tratamientos (Tabla 1). Los tiempos de irradiación según la dosis fueron: 7.08 minutos para 100 Gy, 10.62 minutos para 150 Gy: 14.15 minutos para 200 Gy y 17.69 minutos para 250 Gy. 
Tabla 1. Tratamientos determinados en estudio de pruebas de determinación de DL50 en cebada.

\begin{tabular}{|c|c|}
\hline Tratamiento & Codificación \\
\hline t1 & INIAP-Cañicapa 2003 (testigo) \\
t2 & INIAP-Cañicapa 2003 a 100 Gy \\
t3 & INIAP-Cañicapa 2003 a 150 Gy \\
t4 & INIAP-Cañicapa 2003 a 200 Gy \\
t5 & INIAP-Cañicapa 2003 a 250 Gy \\
t6 & Rita Pelada (testigo) \\
t7 & Rita Pelada a 100 Gy \\
t8 & Rita Pelada a 150 Gy \\
t9 & Rita Pelada a 200 Gy \\
t10 & Rita Pelada a 250 Gy
\end{tabular}

La determinación de la dosis letal media (DL50) en cebada se realizó en dos fases: en laboratorio y en campo. En laboratorio, se determinó el porcentaje de germinación de las semillas irradiadas en cuatro repeticiones: se colocaron 100 semillas por cada tratamiento en papel secante y se dejó en una cámara de germinación a una humedad de $90 \%$ y temperatura de $22^{\circ} \mathrm{C}$. La implementación se realizó en base a un Diseño Completamente al Azar (DCA). A los ocho días después de la siembra se contabilizó el número de semillas germinadas y se las expresó en porcentaje de germinación.

Para la fase de campo, en camas de $7.50 \mathrm{~m}^{2}$ (1.50 m x 5 m), se sembró los diferentes tratamientos, conformado por 50 semillas. La siembra se realizó bajo un Diseño de Bloques Completamente al Azar (DBCA) en cuatro repeticiones. Después de 11 días, se contabilizó la cantidad de semillas germinadas y se lo expresó en porcentaje de emergencia. A los 19 días posteriores a la siembra se determinó la altura de las plántulas en centímetros, desde la base hasta la parte terminal de las mismas; adicionalmente se evaluó de forma visual el vigor de las plántulas por cada tratamiento, empleando una escala cualitativa: $\mathrm{MB}=$ muy bueno, $\mathrm{B}=$ bueno, $\mathrm{R}=$ regular y $\mathrm{M}=$ malo. Con todas las variables evaluadas, se procedió a realizar el análisis de varianza (ANOVA) y la prueba de separación de medias de Tukey al $5 \%$, utilizando para el efecto el programa estadístico InfoStat Versión Profesional 2019.

\section{RESULTADOS}

En el ANOVA (Tabla 2) para porcentaje de germinación en laboratorio, se observó diferencias altamente significativas entre variedades, diferencias significativas entre dosis de irradiación y ninguna significación estadística para repeticiones y la interacción 
variedades por dosis. El promedio general de germinación en laboratorio fue de $82.2 \%$, con un coeficiente de variación de $0.5 \%$, que demuestra la confiabilidad de los resultados obtenidos.

Tabla 2. ANOVA para porcentaje de emergencia en laboratorio en estudio de determinación de DL50 en cebada.

\begin{tabular}{|l|c|c|}
\hline \multicolumn{1}{|c|}{ Fuentes de variación } & Grados de libertad & Cuadrados medios \\
\hline Total & 39 & 57.2 \\
\hline Repeticiones & 3 & $2160.9 * *$ \\
\hline Variedades (V) & 1 & $122.0 *$ \\
\hline Dosis irradiación (D) & 4 & 39.4 n.S. \\
\hline VxD & 4 & 28.4 \\
\hline
\end{tabular}

Promedio: $82.2 \%$

CV: $\quad 0.5 \%$

Nivel de significancia 5\%: $\left(^{* *}\right)$ altamente significativo, $\left({ }^{*}\right)$ significativo, (n.s.) no significativo $\mathrm{CV}$ : coeficiente de variación.

La alta significancia estadística para variedades (Tabla 3), indicó que el porcentaje de germinación no se expresó por igual entre ellas, influyendo las dosis de irradiación. INIAP-Cañicapa 2003 obtuvo un valor de 89.6\% y Rita Pelada un valor de 74.9\% (Fig. 1).

Tabla 3. Prueba Tukey al 5\% para variedades en el porcentaje de emergencia en laboratorio en estudio de determinación de DL50 en cebada.

\begin{tabular}{|c|c|c|}
\hline Variedad & Germinación (\%) & Rangos de significación* \\
\hline INIAP-Cañicapa 2003 & 89.6 & a \\
\hline Rita Pelada & 74.9 & $b$ \\
\hline
\end{tabular}

* Promedios con diferente letra son estadísticamente diferentes $(P<0.05)$. 
Figura 1. Germinación de semillas en laboratorio de dos variedades de cebada en pruebas de determinación de DL50 en cebada.

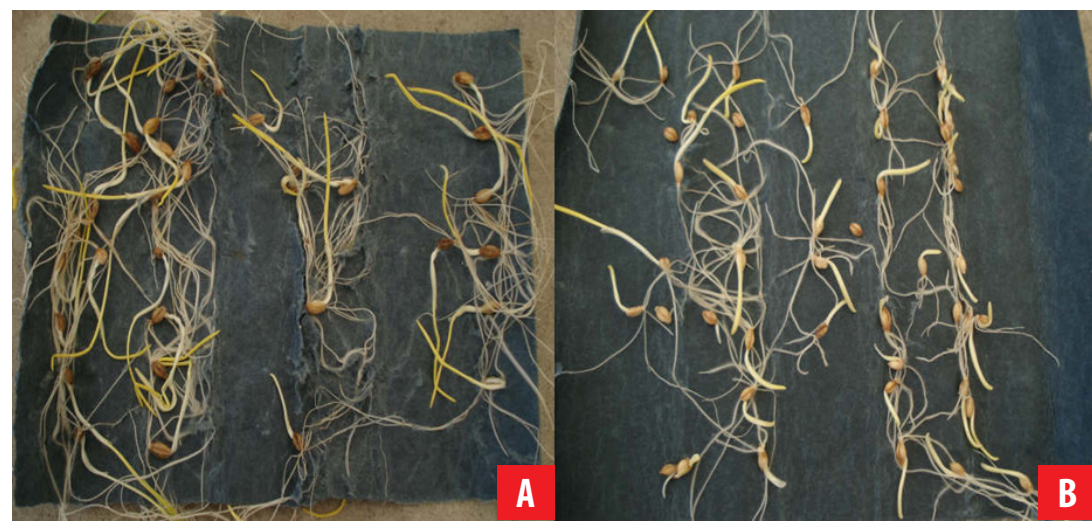

A = INIAP-Cañicapa 2003 y B = Rita Pelada

A pesar de la significancia estadística para el factor dosis de irradiación, la prueba Tukey al 5\%, detectó un solo rango de significación estadística (Tabla 4) con valores entre 79.0 a $88.8 \%$ de porcentaje de germinación.

Tabla 4. Prueba Tukey al $5 \%$ para porcentaje de emergencia en laboratorio por dosis de irradiación en pruebas de determinación de DL50 en cebada.

\begin{tabular}{|c|c|c|}
\hline \multicolumn{1}{|c|}{ Dosis (Gy) } & \multicolumn{2}{c|}{ Rarcentaje de germinación (\%) de significación } \\
\hline 0 & 88.8 & a \\
\hline 100 & 82.5 & a \\
\hline 150 & 81.5 & a \\
\hline 200 & 79.5 & a \\
\hline 250 & 79.0 & a \\
\hline
\end{tabular}

Para las variables de campo, el ANOVA (Tabla 5) para porcentaje de emergencia, se observó diferencias altamente significativas para variedades y dosis y ninguna significancia estadística para repeticiones e interacción variedades por dosis. El promedio para porcentaje de germinación en campo fue de $53.8 \%$, con un coeficiente de variación de 10.8\%, que demuestran la confiabilidad de los resultados obtenidos. 
Tabla 5. ANOVA para porcentaje de emergencia y altura de planta en campo en pruebas de determinación de DL50 en cebada.

\begin{tabular}{|c|c|c|c|c|c|}
\hline \multirow{3}{*}{$\begin{array}{c}\text { Fuentes de variación } \\
\text { Total }\end{array}$} & \multirow{3}{*}{$\begin{array}{c}\text { Grados de libertad } \\
39\end{array}$} & \multicolumn{4}{|c|}{ Cuadrados medios } \\
\hline & & \multicolumn{2}{|c|}{ Emergencia (\%) } & \multicolumn{2}{|c|}{ Altura planta (cm) } \\
\hline & & & & & \\
\hline Repeticiones & 3 & 45.6 & n.s. & 1.4 & n.s. \\
\hline Variedades & 1 & 3027.6 & $* *$ & 240.6 & $* *$ \\
\hline Dosis & 4 & 1455.8 & $* *$ & 28.3 & $* *$ \\
\hline$V \times D$ & 4 & 11.4 & n.s. & 6.5 & $* *$ \\
\hline Error & 27 & 33.7 & & 0.7 & \\
\hline Promedio & & 53.8 & & 8.3 & \\
\hline CV (\%) & & 10.8 & & 10.4 & \\
\hline
\end{tabular}

Nivel de significancia 5\%: $\left(^{* *}\right)$ altamente significativo, $\left(^{*}\right)$ significativo, (n.s.) no significativo. CV: coeficiente de variación.

En la variable porcentaje de emergencia, la prueba Tukey al 5\% para variedades (Tabla 6) detectó dos rangos de significación estadística, ubicándose en el primer rango la variedad INIAP-Cañicapa 2003 con 62.5\% y en el último rango la variedad Rita Pelada con $45.1 \%$

Tabla 6. Prueba Tukey al $5 \%$ para variedades en el porcentaje de germinación en campo en pruebas de determinación de DL50 en cebada.

\begin{tabular}{|c|c|c|}
\hline \multicolumn{2}{|c|}{ Verminación (\%) } \\
\hline INIAP-Cañicapa 2003 & 62.5 & a \\
\hline Rita Pelada & 45.1 & b \\
\hline
\end{tabular}

* Promedios con diferente letra son estadísticamente diferentes $(P<0.05)$.

La prueba Tukey al 5\% para el factor dosis de irradiación (Tabla 7) detectó tres rangos de significación estadística, ubicándose en el primer rango los niveles 0, 100 y 150 Gy con los mejores porcentajes de emergencia: 68.0, 61.5 y 58.5\%, respectivamente. En el segundo rango el nivel de 200 Gy con un valor promedio de $47.2 \%$ de emergencia y en el último rango se ubicó el nivel de 250 Gy con porcentajes de emergencia de 33.8\% (Fig. 1). 
Tabla 7. Tukey al 5\% para dosis de irradiación en porcentaje de germinación en campo en pruebas de determinación de DL50 en cebada.

\begin{tabular}{|c|c|c|c|}
\hline Nivel & Dosis (Gy) & Promedio (\%) & Rango de significación \\
\hline $\mathrm{n} 1$ & 0 & 68.0 & a \\
\hline $\mathrm{n} 2$ & 100 & 58.5 & $a b$ \\
\hline n3 & 150 & 61.5 & a \\
\hline n4 & 200 & 47.2 & $b$ \\
\hline n5 & 250 & 33.8 & c \\
\hline
\end{tabular}

En el ANOVA (Tabla 5), la variable altura de plantas mostró una alta significancia estadística para variedades, dosis de irradiación e interacción variedades por dosis de irradiación; y ninguna significancia estadística para repeticiones. El promedio general de altura de planta fue $8.3 \mathrm{~cm}$ con un coeficiente de variación de 10.4\%.

En la variable altura de planta, la alta significancia estadística para la interacción variedades por dosis, indicó que existe una relación entre los factores en estudio. En la Tabla 8, se observó que la variedad INIAP-Cañicapa 2003 en las diferentes dosis de irradiación presentó valores y rangos altos de altura de planta en comparación a la variedad Rita Pelada, que presentó valores y rangos bajos, debido posiblemente al tipo de grano de cada variedad (Fig. 2).

Tabla 8. Prueba Tukey al $5 \%$ para la interacción variedades por dosis para altura de planta en campo de irradiación en pruebas de determinación de DL50 en cebada.

\begin{tabular}{|c|c|c|c|c|}
\hline Dosis (Gy) & \multicolumn{2}{|c|}{ INIAP-Cañicapa 2003} & \multicolumn{2}{|c|}{ Rita Pelada } \\
\hline 0 & 14.5 & $a^{*}$ & 7.0 & de \\
\hline 100 & 11.8 & $b$ & 7.0 & de \\
\hline 150 & 10.1 & $b c$ & 6.2 & e \\
\hline 200 & 8.5 & $\mathrm{~cd}$ & 5.8 & e \\
\hline 250 & 8.6 & $\mathrm{~cd}$ & 3.1 & $f$ \\
\hline
\end{tabular}




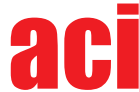

avances
en cienciase en cienciase
ingenierías

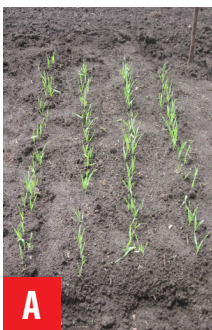

n1 (0 Gy-Testigo)
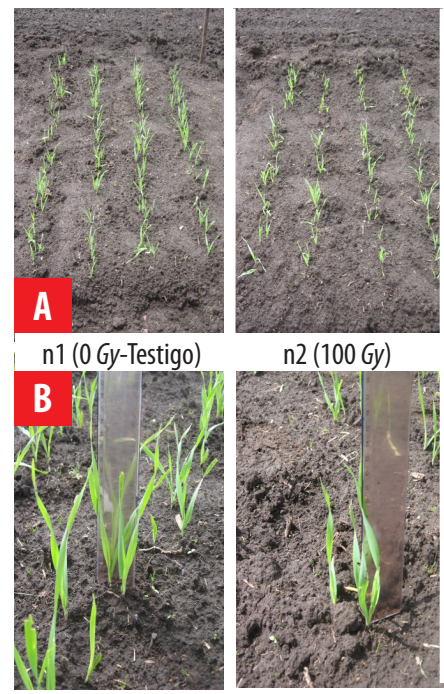

n2 (100 Gy)

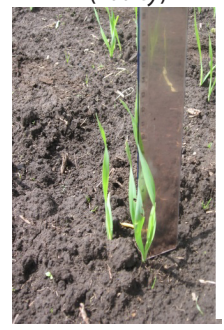

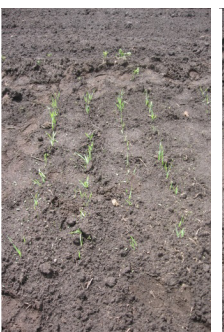

$\mathrm{n} 4(200 \mathrm{~Gy})$
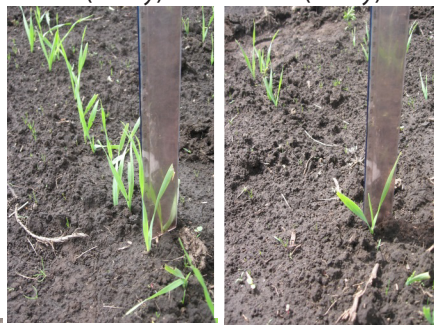

n5 (250 Gy)

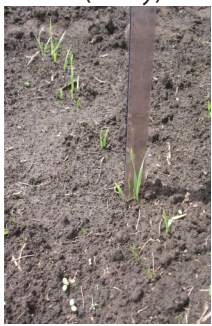

A $=$ Rita Pelada y $B=$ INIAP-Cañicapa 2003

En la variable vigor de planta (Tabla 9), se observa que en las variedades INIAP-Cañicapa 2003 y Rita Pelada, a partir de las dosis de irradiación de 150 Gy a 250 Gy existió una pérdida de vigor de planta, en comparación al testigo (0 Gy).

Tabla 9. Vigor de planta en campo en pruebas de determinación de DL50 de cebada.

\begin{tabular}{|c|c|c|}
\hline \multicolumn{1}{|c|}{ Dosis (Gy) } & Rita Pelada \\
\hline 0 & MB & B \\
\hline 100 & B & B Cañicapa 2003 \\
\hline 150 & B & R \\
\hline 200 & R & M \\
\hline 250 & M & M \\
\hline
\end{tabular}

$\mathrm{MB}=$ muy bueno, $\mathrm{B}=$ bueno, $\mathrm{R}=$ regular $\mathrm{y} \mathrm{M}=$ malo

Con base a los resultados obtenidos, se determinó que la DL50 se encuentra entre los niveles de 150 y 200 Gy, que provocaron daños menores en las plantas, con ligeras diferencias fenotípicas, comparadas con el testigo, tanto en porcentaje de germinación, como en altura de planta y vigor, para las dos variedades. 


\section{DISCUSIÓN}

En el porcentaje de germinación en laboratorio, pese a que no se pudo evidenciar diferencias estadísticas en los promedios, existió una disminución gradual en el porcentaje de germinación al incrementarse la dosis; siendo el tratamiento con el menor promedio la dosis de 250 Gy. De acuerdo a Mayta [20], la disminución en la germinación se debe al daño fisiológico que produce el mutágeno a la semilla y depende mucho de los grados de radio sensibilidad de las especies.

Rozman [21], realizó una investigación en cebada bajo condiciones contraladas y determinó diferencias estadísticas de germinación al utilizar una dosis de 400 Gy 70.5 $\%$ a), mientras que los otros tres tratamientos (testigo, 100 y 200 Gy) no mostraron diferencias estadísticas ( $90.5 \%$, b; $90.6 \%$, b; y $86.8 \%$, b, respectivamente), coincidiendo con los resultados encontrados en el presente estudio [21].

Sin embargo, Sarduie-Nasab, Sharifi-Sirchi, \&Torabi-Sirchi [22], encontraron en su estudio realizado con la variedad de cebada Nosrat, diferencias estadísticas significativas entre los tratamientos frente al testigo, empleando un rango más amplio para las irradiaciones (testigo, 200, 700 y 1200 Gy). Estos resultados, a pesar de ser diferentes variedades, no concuerdan con los obtenidos en este estudio, obteniéndose diferencias significativas con el testigo y la dosis de irradiación con 200 Gy (97\%, a y 66\% b, respectivamente) al determinar la germinación en condiciones de laboratorio [22].

El porcentaje de emergencia observado en todos los tratamientos en campo fue menor al que se obtuvo en condiciones de laboratorio, siendo el tratamiento de $250 \mathrm{~Gy}$, aquel que presentó los menores promedios (33.8\%); los cuales concuerdan con los reportados por Sarduie-Nasab et al. [22], quienes determinaron que los porcentajes de emergencia de semilla irradiada del cultivar de cebada Nosrat, presentaron diferencias significativas entre el testigo y las diferentes dosis de irradiación con rayos gama, siendo comparable a los resultados encontrados en este estudio, donde el testigo presentó un $81 \%$ de emergencia y el tratamiento a 200 Gy un $56 \%$. En el presente estudio, el testigo presentó un 68\% de emergencia y el tratamiento con 200 Gy un $47.2 \%$ [22].

Para la altura de plántula se observó diferencias significativas para las dosis de irradiación, a mayor dosis menor altura de plántula, debido al efecto de la irradiación en las variedades en estudio; lo que concuerda con el trabajo de Sarduie-Nasab et al. [22], que mencionan que a mayor dosis las plantas presentan una disminución en la altura de planta, cantidad de raíces, tamaño y ancho del tallo. Wang, Ma, Cao, Shan, \& Jiao [23] en su estudio con la variedad de cebada de altura "Kunlun 15", irradiadas a diferentes dosis de rayos gamma (testigo, 50, 100, 150, 200, 250 y 300 Gy), indica que a medida que incrementa la dosis de irradiación disminuye la altura en plántula (testigo $14.3 \mathrm{~cm} ; 300 \mathrm{~Gy} 7 \mathrm{~cm}$ ), similares a los datos obtenidos en este estudio con la variedad INIAP Cañicapa (testigo 14.5 cm; 250 Gy 8.6 $\mathrm{cm}$ ) [23]. Varias investigaciones mencionan que la variación en altura no solamente puede presentarse en una disminución del tamaño, sino también puede darse un incremento del crecimiento comparado con el testigo $[20,21,24,25]$.

Con relación al vigor Sarduie-Nasab et al. [22] mencionan que conforme aumenta la dosis de irradiación las plantas muestran características que no favorecen su normal desarrollo, 
afectando su vigor. El mismo trabajo concluye que 200 Gy es la dosis adecuada para irradiar semillas de cebada, resultado muy similar a la dosis identificada en el presente estudio de 150 a 200 Gy. Al respecto Datta [17] menciona que la determinación de la radiosensibilidad y la dosis de DL50 en las etapas de desarrollo de la investigación de mutación varían de un investigador a otro. Varios investigadores informaron diferentes tipos de resultados, por lo expuesto no es aconsejable comenzar el experimento de mutación sobre la base de la literatura disponible. Es mejor hacer un experimento piloto para determinar la dosis óptima.

\section{CONCLUSIONES}

Las pruebas de germinación realizadas en laboratorio no permitieron determinar el nivel de irradiación recomendado para tratar las semillas de cebada; entre tanto que, las pruebas de emergencia en campo permitieron observar diferencias significativas entre los tratamientos, denotando que a mayor dosis de irradiación mayor son los efectos negativos sobre el fenotipo de las variedades.

En laboratorio se obtuvo un porcentaje de germinación promedio de $89.6 \%$ y $74.9 \%$ para INIAP- Cañicapa y Rita Pelada, respectivamente; mientras en campo, INIAP- Cañicapa alcanzó un porcentaje de emergencia promedio de 62.5\% y la variedad Rita Pelada un porcentaje de emergencia promedio de $45.1 \%$.

La variedad Rita Pelada de grano desnudo, presentó la mayor variación fenotípica en campo, tanto en germinación, altura de planta y vigor, especialmente en los niveles n4 (200 Gy) y n5 (250 Gy), respecto al testigo.

La dosis óptima de irradiación según los resultados presentados es 150 Gy, que provocó el menor daño en las plantas, pues no se detectó mayores diferencias fenotípicas, comparado con el testigo, tanto en porcentaje de emergencia, altura y vigor, para las dos variedades estudiadas.

\section{RECOMENDACIONES}

Con los resultados obtenidos, se recomienda utilizar la dosis de 150 Gy (n3) para irradiar las semillas de las dos variedades de cebada empleadas en este trabajo. Adicionalmente, al momento de formar la población $\mathrm{M}_{1}$, ajustar la densidad de siembra para las dos variedades objeto del estudio, tomando en cuenta los porcentajes de germinación obtenidos, con el fin de obtener la cantidad de individuos necesarios, 50000 plantas, en la población a evaluar.

\section{AGRADECIMIENTOS}

Los autores agradecen por su apoyo al: Organismo Internacional de Energía (OIEA) con los proyectos: ECU/5/023 "Inducing Mutations in Agriculture with the Aid of Radiation", y RLA/5/068 ": Improving Yield and Commercial Potential of Crops of Economic 
Importance (ARCAL CL)". A la Subsecretaría de Control y Aplicaciones Nucleares (SCAN) del Ministerio de Energía y Recursos Naturales No Renovables (MERNNR), por las facilidades prestadas para el uso del irradiador tipo I, ubicado en las instalaciones de Aychapicho, y al Instituto Nacional de Investigaciones Agropecuarias (INIAP) por todo el apoyo recibido en la investigación.

\section{CONTRIBUCIONES DE LOS AUTORES}

Javier Garófalo y Luis Ponce propusieron la investigación, evaluaron, registraron y analizaron los datos de laboratorio y campo; Javier Garófalo redactó el manuscrito; Luis Ponce, Patricio Noroña y Diego Campaña proveyeron bibliografía, aportaron en la discusión y análisis de los resultados. 


\section{REFERENCIAS}

[1] Falconí, E., Garófalo, J., Llangarí, P., \& Espinoza, M. (2013). El cultivo de cebada: Guía para la producción de semilla de calidad. Instituto Nacional de Investigaciones Agropecuarias, Boletín Divulgativo N 390. Quito, Ecuador.

[2] Ministerio de Agricultura y Ganadería. (2019). Cifras Agroproductivas 2019. Recuperado de http://sipa.agricultura. gob.ec/index.php/cifras-agroproductivas. Consultado septiembre 2020.

[3] Instituto Nacional de Investigaciones Agropecuarias. (2010). Convenio de Cooperación Técnica Interinstitucional Corpoiniap y Cervecería Nacional. Quito, Ecuador.

[4] Ponce-Molina, L., Garófalo, J., Campaña, D. Noroña, P. (2019). Parámetros de Evaluación y Selección en Cereales. Instituto Nacional de Investigaciones Agropecuarias. Manual № 116. Quito, Ecuador.

[5] Ponce-Molina, L., Noroña, P., Campaña, D., Garófalo, J., Coronel, J., Jiménez, C., \& Cruz, E. (2020). La Cebada (Hordeum vulgare L.): Generalidades y variedades mejoradas para la Sierra Ecuatoriana. Instituto Nacional de Investigaciones Agropecuarias. Quito, Ecuador.

[6] Ahloowalia, B. S., \& Maluszynski, M. (2001). Induced mutations - A new paradigm in plant breeding. Euphytica, 118(2), 167-173. Doi: https://doi.org/10.1023/A:1004162323428

[7] Irfaq, M., \& Nawab, K. (2001). Effect of Gamma Irradiation on Some Morphological Characteristics of Three Wheat (Triticum aestivum L.) Cultivars. Journal of Biological Sciences, 1(10), 935-937. Doi: https://doi.org/10.3923/ jbs.2001.935.937

[8] Soraluz Cubas, L. E. (2015). Inducción de mutaciones en centeno (Secale cereale Linneo) empleando radiación gamma. Universidad Nacional Agraria La Molina. Recuperado de http://repositorio.lamolina.edu.pe/handle/UNALM/1421. Consultado agosto 2020.

[9] Solano S., J., Barriga B., P., Krarup H., A., \& Figueroa S., H. (1998). Estabilidad Temporal Del Rendimiento De Genotipos Mutantes De Trigo, Mediante El Modelo De Interaccion Multiplicativa Y Efectos Principales Aditivos (Ammi: Additive Main Effect and Multiplicative Interaction Model). Agro Sur, 26(2), 19-32. Doi: https://doi.org/10.4206/agrosur.1998. v26n2-03

[10] Hernández Muñoz, S., Pedraza Santos, M. E., López, P. A., De la Cruz Torres, E., Fernández Pavía, S. P., Martínez Palacios, A., \& Martínez Trujillo, M. (2017). Determinación de la DL 50 y GR 50 con rayos gamma $(60 \mathrm{Co})$ en protocormos de Laelia autumnalis in vitro. Agrociencia, 51, 507-524.

[11] Gupta, S., Kumar Datta, A., Pramanik, A., Biswas, J., \& Karmakar, R. (2019). X-ray and gamma irradiation Induced chromosomal Aberrations in plant species as the consequence of induced mutagenesis - an overview. Plant Archives, 19(2), 1973-1979.

[12] Ulukapi, K., \& Nasircilar, A. G. (2015). Developments of Gamma Ray Application on Mutation Breeding Studies in Recent Years. International Conference on Advances in Agricultural, Biological \& Environmental Sciences. London. Doi: https://doi.org/10.15242/iicbe.c0715044

[13] Di Pane, F. J., Lopez, S. C., Cantamutto, M. Á., Domenech, M. B., \& Castro-Franco, M. (2018). Effect of different gamma radiation doses on the germination and seedling growth of wheat and triticale cultivars. Australian Journal of Crop Science, 12(12), 1921-1926. Doi: https://doi.org/10.21475/ajcs.18.12.12.p1251

[14] Cheema, A. Al., \& Manzoor Atta, B. (2003). RADIOSENSITIVITY STUDIES IN BASMATI RICE. Pak. J. Bot., 35(2), 197-207.

[15] Jain Mohan, S. (2005). Major mutation-assisted plant breeding programs supported by FAO / IAEA. Plant Cell, Tissue and Organ Culture, 82, 113-123.

[16] Cortés Conget, C. A. (2017). Estimación de dosis letal 50 en dos explantes de Alstroemeria spp. irradiados con rayos gamma (Tesis de pre grado). Universidad de Chile, Santiago.

[17] Datta, S. K: (2019). Determination of Radiosensitivity : Prerequisite Factor for Induced Mutagenesis. En C. P. Malik and P.C. Trivedi, Harnessing Plant Biotechnology and Physiology to Stimulate Agricultural Growth. Agrobios, India.

[18] Bhan, A. K. (1979). Towards a more efficient mutation breeding programme in Barley. Genetics Newsletter, 3(II), $11-12$. 
[19] Mathías R., M., \& Fernández, F. (2019). Recomendaciones para reducir el riesgo de tendedura en avena. Biblioteca Digital. Santiago de Chile.

[20] Mayta, M. E. (2016). Dosimetría de Rayos Gamma para la Inducción de Mutación en Cañihua (Chenopodium pallidicaule Aellen ) (Tesis de pre grado Universidad Nacional Agraria La Molina). Lima.

[21] Rozman, L. (2014). The effect of gamma radiation on seed germination of barley (Hordeum vulgare $\mathrm{L}$.). Acta agriculturae Slovenica, 103(2), 307-311. Doi: https://doi.org/10.14720/aas.2014.103.2.15

[22] Sarduie-Nasab, S., Sharifi-Sirchi, G. R., \& Torabi-Sirchi, M. H. (2010). Assessment of dissimilar gamma irradiations on barley (Hordeum vulgare). Journal of Plant Breeding and Crop Science, 2(4), 59-063.

[23] Wang, X., Ma, R., Cao, Q., Shan, Z., \& Jiao, Z. (2018). Enhanced tolerance to salt stress in highland barley seedlings ( Hordeum vulgare ssp . vulgare ) by gamma irradiation pretreatment. Acta Physiologiae Plantarum, 40(174), 1-11. Doi: https://doi.org/10.1007/s11738-018-2736-2

[24] Geras'kin, S., Churyukin, R., \& Volkova, P. (2017). Radiation exposure of barley seeds can modify the early stages of plants' development. Journal of environmental radioactivity, 177, 71-83. Doi: https://doi.org/10.1016/j. jenvrad.2017.06.008

[25] Obare, I. J., Kinyua, M. G., \& Kiplagat, 0. K. (2014). Effects of Induced Mutagenesis on Agronomic Traits in Barley (Hordeum vulgare L.). American Journal of Experimental Agriculture, 4(12), 1536-1543. Doi: https://doi.org/10.9734/ AJEA/2014/1115 